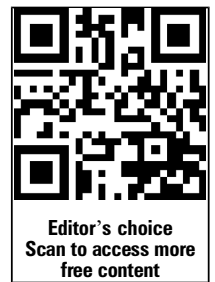

For numbered affiliations see end of article.

\section{Correspondence to} Dr Jan Minners, Department of Cardiology, University Hospital Basel, Petersgraben 4, CH-4031 Basel, Switzerland; jminners@uhbs.ch

Received 12 June 2013 Revised 25 July 2013 Accepted 29 July 2013 Published Online First 22 August 2013

\section{SLinked}

- http://dx.doi.org/10.1136/ heartjnl-2013-304747

To cite: Jander N, GohlkeBärwolf C, Bahlmann E, et al. Heart 2014;100: 28-33.

\title{
Indexing aortic valve area by body surface area increases the prevalence of severe aortic stenosis
}

\author{
Nikolaus Jander, ${ }^{1}$ Christa Gohlke-Bärwolf, ${ }^{1}$ Edda Bahlmann, ${ }^{2}$ Eva Gerdts, ${ }^{3}$ \\ Kurt Boman, ${ }^{4}$ John B Chambers, ${ }_{1}^{5}$ Kenneth Egstrup, ${ }^{6}$ Christoph A Nienaber, ${ }^{7}$ \\ Terje R Pedersen, ${ }^{8}$ Simon Ray, ${ }_{1}^{9}$ Anne B Rossebø, ${ }^{10}$ Ronnie Willenheimer, ${ }^{11}$ \\ Rolf-Peter Kienzle, ${ }^{1}$ Kristian Wachtell, ${ }^{12}$ Franz-Josef Neumann, ${ }^{1}$ Jan Minners ${ }^{13}$
}

ABSTRACT

Background To account for differences in body size in patients with aortic stenosis, aortic valve area (AVA) is divided by body surface area (BSA) to calculate indexed AVA ( $\left.A V A_{\text {index }}\right)$. Cut-off values for severe stenosis are $<1.0 \mathrm{~cm}^{2}$ for AVA and $<0.6 \mathrm{~cm}^{2} / \mathrm{m}^{2}$ for $\mathrm{AVA}_{\text {index }}$. Objective To investigate the influence of indexation on the prevalence of severe aortic stenosis and on the predictive accuracy regarding clinical outcome.

Methods Echocardiographic and anthropometric data from a retrospective cohort of 2843 patients with aortic stenosis (jet velocity $>2.5 \mathrm{~m} / \mathrm{s}$ ) and from 1525 patients prospectively followed in the simvastatin and ezetimibe in aortic stenosis (SEAS) trial were analysed.

Results The prevalence of severe stenosis increased with the $\mathrm{AVA}_{\text {index }}$ criterion compared to AVA from $71 \%$ to $80 \%$ in the retrospective cohort, and from $29 \%$ to $44 \%$ in SEAS (both $p<0.001$ ). Overall, the predictive accuracy for aortic valve events was virtually identical for AVA and AVA $_{\text {index }}$ in the SEAS population (mean follow-up of 46 months; area under the receiver operating characteristic curve: $0.67(95 \% \mathrm{Cl} 0.64$ to 0.70 ) vs 0.68 (Cl 0.65 to 0.71) (NS). However, 213 patients additionally categorised as severe by $\mathrm{AVA}_{\text {index }}$ experienced significantly less valve related events than those fulfilling only the AVA criterion $(p<0.001)$.

Conclusions Indexing AVA by BSA ( AVA $_{\text {index }}$ ) significantly increases the prevalence of patients with criteria for severe stenosis by including patients with a milder degree of the disease without improving the predictive accuracy for aortic valve related events.

\section{INTRODUCTION}

Indexing aortic valve area (AVA) by body surface area (BSA) $\left(\mathrm{AVA}_{\text {index }}\right)$ represents an intuitively convincing and widely adopted method to adjust for differences in body size. ${ }^{1-3}$ Whereas current American College of Cardiology/American Heart Association guidelines on valvular heart disease recommend indexing AVA for all patients, ${ }^{2}$ the recently published European Society of Cardiology (ESC) guidelines state that indexing 'may be helpful, particularly in patients with an unusually small BSA'. ${ }^{1}$ In the European Association of Echocardiography/American Society of Echocardiography recommendations for clinical practice, the role of indexing is considered controversial particularly because of the uncertain role of excess body weight. ${ }^{4}$
Cut-off values for severe stenosis were set in all guidelines at $<1.0 \mathrm{~cm}^{2}$ for AVA and $<0.6 \mathrm{~cm}^{2} / \mathrm{m}^{2}$ for $\mathrm{AVA}_{\text {index }}$. However, clinical or outcome studies supporting this recommendation are rare. ${ }^{5}$ In theory, calculation of $\mathrm{AVA}_{\text {index }}$ should not change the overall number of patients with severe stenosis in a given population but rather result in a decrease in the percentage of patients with the diagnosis of severe stenosis in smaller patients and an increase in larger patients. Based on the hypothesis that adjusting AVA by BSA, that is, AVA $_{\text {index }}$, improves the comparability of stenosis severity in patients with diverging body size we investigated the impact of $\mathrm{AVA}_{\text {index }}$ on the prevalence of severe aortic stenosis and the prediction of clinical events compared to (unindexed) AVA in two large populations of patients with aortic valve stenosis and normal left ventricular function.

\section{PATIENTS AND METHODS}

Data from two distinct study populations are presented. First, we identified 2843 consecutive patients investigated at the echocardiographic laboratory of the Heart Centre Bad Krozingen between January 1998 and December 2010 demonstrating normal left ventricular function and a jet velocity of $\geq 2.5 \mathrm{~m} / \mathrm{s}$. Patients with a moderate or severe mitral or aortic regurgitation, a mean pressure gradient $<10 \mathrm{~mm} \mathrm{Hg}$ or incomplete data were excluded. Normal left ventricular function was defined as normal global systolic left ventricular function with fractional shortening $\geq 30 \%$ without regional wall motion abnormalities.

The second cohort was recruited from the prospective SEAS study (NCT00092677), which enrolled 1873 patients with asymptomatic aortic stenosis, defined by echocardiography at local study centres as aortic valve thickening and transaortic Doppler jet velocity $\geq 2.5$ and $\leq 4.0 \mathrm{~m} / \mathrm{s}$. Patients were randomised from January 2001 to February 2004 to at least 4-year placebo-controlled combined treatment with ezetimibe $10 \mathrm{mg} /$ day and simvastatin $40 \mathrm{mg} /$ day. Patients with coronary heart disease, heart failure, diabetes, history of stroke or peripheral vascular disease, clinically significant mitral valve disease, severe or predominant aortic regurgitation, rheumatic valvular disease, aortic valve prosthesis or renal insufficiency, and patients already on lipid-lowering therapy or having an indication for lipid lowering according to guidelines 
were excluded. The original SEAS study showed no difference in major cardiovascular events between treatment and placebo group but a significant reduction in ischaemic events in patients treated with ezetimibe/simvastatin. ${ }^{6}$ The present study population comprises $1525(81.4 \%)$ of the 1873 patients recruited in the SEAS trial with an ejection fraction $\geq 55 \%$ in whom a detailed echocardiographic assessment by the core laboratory at baseline was available.

Echocardiography was performed following the guidelines for the clinical application of echocardiography ${ }^{48}$ and has been described in detail elsewhere. $69{ }^{10}$ Severe aortic stenosis was defined according to current guidelines ${ }^{1}{ }^{2}$ by an AVA $<1.0 \mathrm{~cm}^{2}$ and an $\mathrm{AVA}_{\text {index }}<0.6 \mathrm{~cm}^{2} / \mathrm{m}^{2}$. The velocity ratio (VR) as a body size independent measure of aortic stenosis severity was calculated by the velocity time integral (VTI) of pulsed wave Doppler in the left ventricular outflow tract divided by the VTI of continuous Doppler through the aortic valve. Anthropometric data (height and weight) were recorded electronically at the time of echocardiography. BSA was calculated from the Mosteller formula ${ }^{11}:$ BSA $=\sqrt{(\text { height } \times \text { weight }) / 3600}$. Body mass index (BMI) was calculated as $\mathrm{BMI}=$ weight $/$ height $^{2}$. The primary end point of the outcome analysis of the present study was aortic valve related events (defined as aortic valve replacement, congestive heart failure due to aortic stenosis, or death from cardiovascular causes); the secondary end point was cardiovascular death. All end points had been adjudicated with a predefined end point protocol by an end point committee blinded for study conduct and results in the original SEAS study. ${ }^{6}$ All statistics were performed using SPSS V.15.0 with continuous variables presented as mean $\pm \mathrm{SD}$ and categorical variables as percentages. Percentages were compared with the use of the Pearson $\chi^{2}$ test. Receiver operating characteristic (ROC) curves were constructed to assess the sensitivity and specificity of parameters of AVA and an $\mathrm{AVA}_{\text {index }}$ and to compare their ability to predict aortic valve events or cardiovascular death. The comparison of areas under the ROC curves (AUC) was performed as recommended by DeLong et al. ${ }^{12}$ The Kaplan-Meier method was used to assess event-free survival with differences checked by means of the log-rank test. All testing was two-tailed, and $\mathrm{p}$ values of less than 0.05 were considered to indicate statistical significance.

\section{RESULTS}

Clinical, anthropometric and echocardiographic data of both the retrospective cohort from our centre including 2843 patients and the prospectively followed SEAS population $(n=1525)$ are summarised in table 1 . Whereas the retrospective cohort corresponds to the typically encountered patients with a wide range of aortic stenosis severity, including very severe stenosis, referred to a tertiary care centre, the SEAS population represents selected patients with low cardiovascular risk and predominantly mild to moderate stenosis.

\section{Prevalence of severe aortic valve stenosis according to AVA and AVA $_{\text {index }}$}

Indexation by BSA increased the prevalence of severe stenosis in the retrospective cohort from $71 \%\left(\mathrm{AVA}<1.0 \mathrm{~cm}^{2}\right)$ to $80 \%$ $\left(\mathrm{AVA}_{\text {index }}<0.6 \mathrm{~cm} / \mathrm{m}^{2}, \mathrm{p}<0.001\right)$. In the prospective SEAS population there was a similar increase from $31 \%$ to $44 \%$ $(\mathrm{p}<0.001)$. The percentages related to gender are given in table 2 .

To study the impact of indexation on patients with diverging body size, we stratified the populations according to BSA quartiles demonstrating a body size dependency of AVA in both populations to the effect that larger patients had a lower prevalence of severe stenosis (figure $1 \mathrm{~A}, \mathrm{~B}, \mathrm{p}$ for trend $<0.001$ ) whereas $\mathrm{AVA}_{\text {index }}$ was independent of BSA. Compared to AVA, AVA ${ }_{\text {index }}$ did not significantly decrease the percentage of patients with severe stenosis in small patients (BSA $\leq 1.75 \mathrm{~m}^{2}: 81 \%$ vs $84 \%$, NS, figure $1 \mathrm{~A}$ ), but augmented it in the three remaining quartiles in the retrospective cohort (BSA 1.76-1.88 $\mathrm{m}^{2}$ : $81 \%$ vs $75 \%$; BSA $1.89-2.03 \mathrm{~m}^{2}$ : $77 \%$ vs $67 \%$; BSA $>2.03 \mathrm{~m}^{2}: 80 \%$ vs $58 \%$, all $\left.\mathrm{p}<0.001\right)$. A similar pattern was found in the SEAS population $\left(\mathrm{AVA}_{\text {index }} \mathrm{vs}\right.$ AVA: BSA $\leq 1.76 \mathrm{~m}^{2}: 43 \%$ vs $45 \%$; BSA $1.77-1.89 \mathrm{~m}^{2}: 43 \%$ vs $33 \%$; BSA $1.90-2.03 \mathrm{~m}^{2}$ : 44\% vs $27 \%$; BSA $>2.03 \mathrm{~m}^{2}: 45 \%$ vs $18 \%$, all $\mathrm{p}<0.001$ except $\mathrm{AVA}_{\text {index }}$ vs AVA: BSA $\leq 1.76 \mathrm{~m}^{2}$, NS, figure 1B). Calculation of the body size independent VR confirmed

Table 1 Clinical, anthropometric and echocardiographic characteristics

\begin{tabular}{|c|c|c|c|c|c|c|}
\hline & \multicolumn{3}{|c|}{ Retrospective cohort $(n=2843)$} & \multicolumn{3}{|c|}{ SEAS cohort $(n=1525)$} \\
\hline & Mean & SD & Range & Mean & SD & Range \\
\hline Age (years) & 73.1 & 10.2 & $21-97$ & 67.4 & 9.6 & $28-85$ \\
\hline Female $(\%)$ & 48 & & & 39 & & \\
\hline Height $(\mathrm{cm})$ & 167.4 & 8.9 & $140-198$ & 170.5 & 9.2 & 145-199 \\
\hline Weight $(\mathrm{kg})$ & 77.2 & 14.9 & 39-159 & 78.1 & 14.2 & $42-159$ \\
\hline Body surface area $\left(\mathrm{m}^{2}\right)$ & 1.89 & 0.2 & $1.3-2.8$ & 1.91 & 0.2 & $1.4-2.9$ \\
\hline Body mass index $\left(\mathrm{kg} / \mathrm{m}^{2}\right)$ & 27.5 & 4.5 & $15.6-51.9$ & 26.8 & 4.2 & $15.5-51.6$ \\
\hline LV end-diastolic diameter (mm) & 47.4 & 5.4 & $28-68$ & 50.2 & 6.1 & $26-72$ \\
\hline LV end-systolic diameter (mm) & 28.5 & 4.9 & $11-46$ & 31.6 & 5.3 & $16-52$ \\
\hline Shortening fraction (\%) & 40.1 & 6.5 & $30-68$ & 37.2 & 5.9 & $20-58$ \\
\hline Ejection fraction (Teichholz, \%) & 64.8 & 7.6 & $51-91$ & 66.4 & 7.5 & $40-88$ \\
\hline LVOT diameter (mm) & 20.5 & 2.1 & $14-31$ & 21.9 & 2.6 & $14-32$ \\
\hline LVOT flow velocity $(\mathrm{m} / \mathrm{s})$ & 1.04 & 0.18 & $0.7-1.9$ & 0.97 & 0.20 & $0.5-1.8$ \\
\hline Jet velocity $(\mathrm{m} / \mathrm{s})$ & 4.1 & 0.9 & $2.5-6.7$ & 3.1 & 0.5 & $1.7-4.8$ \\
\hline Mean pressure gradient $(\mathrm{mm} \mathrm{Hg})$ & 40.4 & 17.6 & 10-117 & 22.9 & 8.8 & $6-64$ \\
\hline Aortic valve area $\left(\mathrm{cm}^{2}\right)$ & 0.89 & 0.32 & $0.3-2.3$ & 1.28 & 0.46 & $0.4-4.0$ \\
\hline Aortic valve area index $\left(\mathrm{cm}^{2} / \mathrm{m}^{2}\right)$ & 0.47 & 0.16 & $0.2-1.1$ & 0.67 & 0.23 & $0.2-1.9$ \\
\hline Velocity ratio & 0.27 & 0.08 & $0.1-0.5$ & 0.34 & 0.10 & $0.1-0.8$ \\
\hline
\end{tabular}


Table 2 Prevalence of severe aortic stenosis according to AVA and $\mathrm{AVA}_{\text {index }}$

\begin{tabular}{|c|c|c|c|c|c|}
\hline & \multicolumn{2}{|c|}{$A V A<1.0 \mathrm{~cm}^{2}$} & \multicolumn{2}{|c|}{ AVAindex $<0.6 \mathrm{~cm}^{2} / \mathrm{m}^{2}$} & \multirow[b]{2}{*}{ p Value } \\
\hline & $\mathrm{N}$ & $\%$ & $\mathbf{n}$ & $\%$ & \\
\hline \multicolumn{6}{|c|}{ Retrospective cohort $(n=2843)$} \\
\hline All patients & 2011 & 70.7 & 2268 & 79.8 & $<0.001$ \\
\hline Male $n=1492$ & 904 & 60.6 & 1118 & 74.9 & $<0.001$ \\
\hline Female $n=1351$ & 1107 & 81.9 & 1150 & 85.1 & 0.03 \\
\hline \multicolumn{6}{|c|}{ SEAS cohort $(n=1525)$} \\
\hline All patients & 470 & 30.8 & 668 & 43.8 & $<0.001$ \\
\hline Male $n=931$ & 214 & 23.0 & 392 & 42.1 & $<0.001$ \\
\hline Female $n=594$ & 256 & 43.1 & 276 & 46.5 & n.s. \\
\hline
\end{tabular}

Prevalence of severe aortic valve stenosis defined by the criterion $A V A_{\text {index }}<0.6 \mathrm{~cm}^{2} / \mathrm{m}^{2}$ is increased compared to AVA $<1.0 \mathrm{~cm}^{2}$.

AVA, aortic valve area; $\mathrm{AVA}_{\text {index, }}$ aortic valve area indexed to body surface area; SEAS, simvastatin and ezetimibe in aortic stenosis trial. ${ }^{6}$

comparable stenosis severity between quartiles (mean VR in the retrospective cohort: $\mathrm{BSA} \leq 1.75 \mathrm{~m}^{2}: \quad 0.26 \pm 0.1$; $\mathrm{BSA}$ 1.76-1.88 $\mathrm{m}^{2}: 0.26 \pm 0.1$; BSA $1.89-2.03 \mathrm{~m}^{2}: 0.27 \pm 0.1$; BSA $>2.03 \mathrm{~m}^{2}: 0.27 \pm 0.1$; mean VR in the SEAS study: BSA $\leq 1.76 \mathrm{~m}^{2}$ :
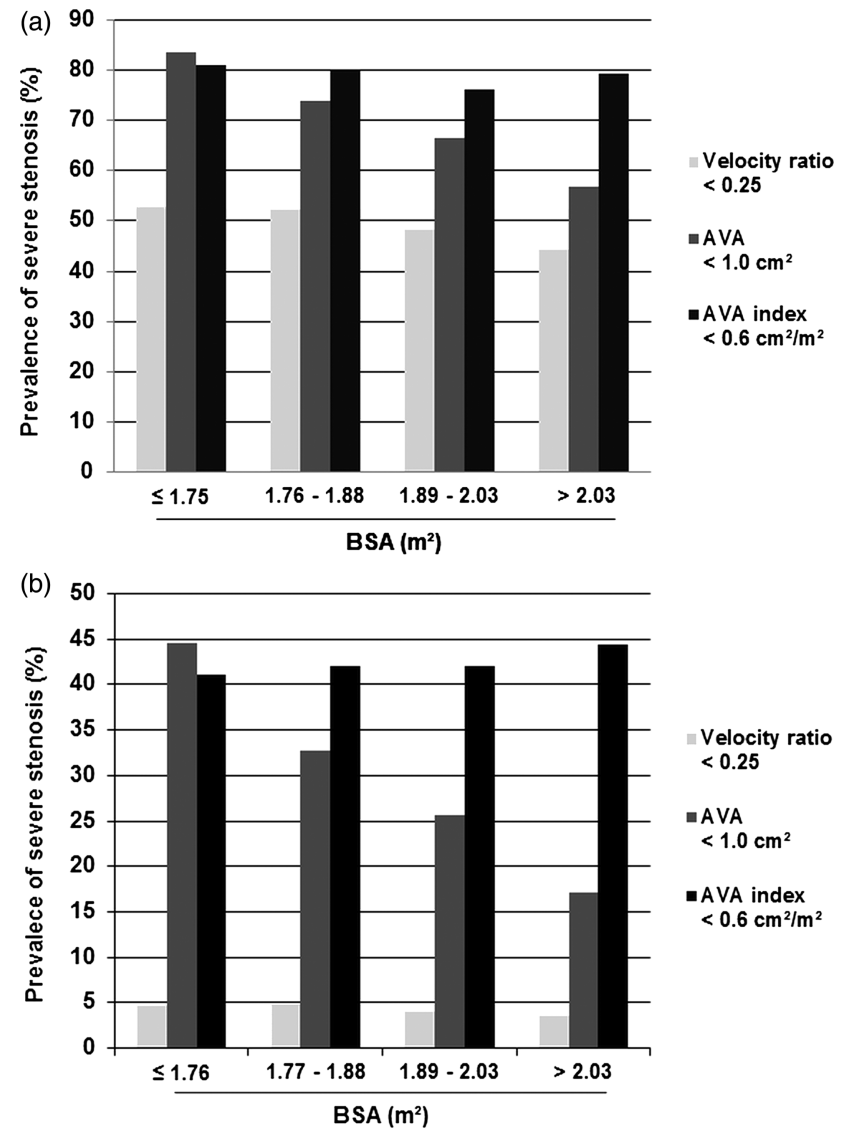

Figure 1 Prevalence of severe aortic valve stenosis after stratification according to body surface area (BSA). The prevalence of patients with severe stenosis according to the aortic valve area (AVA) criterion $\left(<1.0 \mathrm{~cm}^{2}\right)$ is body size dependent in both the retrospective cohort (a) and the simvastatin and ezetimibe in aortic stenosis population (b), with a higher prevalence of severe stenosis in smaller patients.

Calculation of $\mathrm{AVA}_{\text {index }}$ decreases body size dependency but increases the overall prevalence of severe stenosis compared to AVA $(p<0.001)$ in all quartiles except in small patients (BSA $\left.\leq 1.75 / 1.76 \mathrm{~m}^{2}\right)$. The percentage of severe aortic stenosis according to velocity ratio is given for comparison.
$0.33 \pm 0.1 ;$ BSA $1.77-1.89 \mathrm{~m}^{2}: 0.33 \pm 0.1 ;$ BSA $1.90-2.03 \mathrm{~m}^{2}$ : $0.34 \pm 0.1$; BSA $>2.03 \mathrm{~m}^{2}: 0.34 \pm 0.1$ ).

Indexation has been recommended specifically in very small patients with a $\mathrm{BSA}<1.5 \mathrm{~m}^{2}$ or a $\mathrm{BMI}<22 .{ }^{45}$ In our study patients with a BSA $<1.5 \mathrm{~m}^{2}$ were classified with severe stenosis in $95 \%$ before and $85 \%$ after indexation in the retrospective cohort $(\mathrm{n}=91, \mathrm{p}<0.04)$ and in $47 \%$ vs $37 \%$ in SEAS $(\mathrm{n}=19, \mathrm{p}=0.34)$. In patients with a $\mathrm{BMI}<22$ indexation had no statistically significant effect on the prevalence of severe stenosis (retrospective cohort $(\mathrm{n}=228): 80 \%$ vs $77 \%, \mathrm{p}=0.47$, SEAS $(\mathrm{n}=154): 37 \%$ vs $40 \%$, $\mathrm{p}=0.34)$. On the other hand and to exclude a potential influence of excess body weight, ${ }^{4}$ we repeated the analysis with the exclusion of obese patients (BMI $\geq 30$ ) resulting in a mean BSA of $1.83 \mathrm{~m}^{2}$ and $1.86 \mathrm{~m}^{2}$ for the retrospective and the SEAS cohort, respectively. The pattern of increasing the prevalence of severe aortic stenosis in the two cohorts (overall retrospective cohort $(n=2128)$ : $\mathrm{AVA}_{\text {index }} 78 \%$ vs AVA $72 \%$; overall SEAS ( $\left.\mathrm{n}=1227\right)$ : $42 \%$ vs $31 \%$, both $\mathrm{p}<0.001$ ) as well as in the predefined BSA quartiles (data not shown) remained identical. When considering only normal weighed patients (BMI 18.5-24.9), severe stenosis was diagnosed according to $\mathrm{AVA}_{\text {index }}$ in $42.8 \%$ and according to AVA in $34.9 \%$ in the SEAS cohort $(\mathrm{p}=0.01, \mathrm{n}=538)$. The corresponding values for the retrospective cohort $(n=889)$ were $76.6 \%$ versus $74.4 \%$ which was no longer statistically significant $(\mathrm{p}=0.29)$.

\section{Inconsistent grading}

Indexation has also been recommended specifically in patients with 'inconsistent grading' (mean pressure gradient $\leq 40 \mathrm{~mm} \mathrm{Hg}$, AVA $<1.0 \mathrm{~cm}^{2}$ or, inversely, mean pressure gradient $>40 \mathrm{~mm} \mathrm{Hg}$ and AVA $\geq 1.0 \mathrm{~cm}^{2}$ ) in order to reduce discrepancies in the assessment of stenosis severity by gradients and AVA. ${ }^{13}$ However, based on the cut-off value for severe stenosis of $\mathrm{AVA}_{\text {index }} 0.6 \mathrm{~cm}^{2} / \mathrm{m}^{2}$ (and mean pressure gradient $40 \mathrm{~mm} \mathrm{Hg}$ ) the overall number of 'inconsistently graded' patients increased rather than decreased after indexation in both populations (retrospective cohort: from $26 \%$ to $32 \%$; SEAS: from $30 \%$ to $42 \%$; both $\mathrm{p}<0.001$ ) and was unchanged even in the quartile with the smallest patients (retrospective cohort: $33 \%$ vs $31 \%$; SEAS study $42 \%$ vs $41 \%$; both NS). Adjusting the cut-off to $0.5 \mathrm{~cm}^{2} / \mathrm{m}^{2}$ as recently suggested by Michelena $e t a l^{14}$ significantly decreased inconsistent grading in both populations (retrospective cohort: from $32 \%$ to $23 \%$; SEAS: from $42 \%$ to $24 \%$, both $\mathrm{p}<0.001$ ).

Patient outcome according to AVA and AVA index $_{\text {. }}$

In the prospectively followed cohort from SEAS $(n=1525)$ there were 495 aortic valve events, consisting of 431 aortic valve 
replacements, 21 heart failures due to progression of aortic stenosis and 43 cardiovascular deaths over a mean follow-up of 45.8 \pm 14.1 months. Predictive accuracy for aortic valve events was unchanged by indexation as quantified by the AUC (AUC for AVA: 0.67 (95\% CI 0.64 to 0.70$)$; AUC for $\mathrm{AVA}_{\text {index }}$ : 0.68 (CI 0.65 to 0.71 ); NS; figure $2 \mathrm{~A}$ ). When excluding aortic valve replacement from the combined endpoint, AVA was superior to $\mathrm{AVA}_{\text {index }}$ in predicting the remaining 64 events of cardiovascular death and heart failure due to progression of aortic stenosis (AUC for AVA: 0.61 (CI 0.58 to 0.63 ) vs $\mathrm{AVA}_{\text {index }}$ : 0.58 (CI 0.55 to 0.60$), p<0.01)$. There was no difference in predictive accuracy with respect to cardiovascular death alone between the two parameters of aortic valve stenosis severity (AUC for AVA: 0.61 (CI 0.58 to 0.63 ); AUC for $\mathrm{AVA}_{\text {index }}$ : 0.60 (CI 0.57 to 0.62 ), NS; figure $2 \mathrm{~B}$ ). To further analyse the effect of indexation on rates of clinical outcome we compared clinical events between the 470 patients with severe stenosis according to AVA with 213 patients additionally categorised as severe according and limited to the $\mathrm{AVA}_{\text {index }}$ criterion. The latter were more often male, younger and had a higher BSA (all $\mathrm{p}<0.001$ ). Over a mean follow-up of 46 months, outcome in the 213 patients was significantly better with respect to aortic valve related events (figure $3 \mathrm{~A}, \mathrm{p}<0.001$ ) with a trend towards a lower cardiovascular mortality (figure $3 \mathrm{~B}$, $\mathrm{p}=0.09$ ), indicating that indexing AVA by BSA and applying a cut-off value of $0.6 \mathrm{~cm}^{2} / \mathrm{m}^{2}$ increases the prevalence of severe aortic stenosis by including individuals with a milder degree of the disease.

\section{DISCUSSION}

Our data from two large, distinct cohorts totalling 4368 patients with a wide range of aortic valve stenosis severity indicate that indexing AVA by BSA $\left(\mathrm{AVA}_{\text {index }}\right)$ and applying the current partition value of $0.6 \mathrm{~cm}^{2} / \mathrm{m}^{2}$ significantly increases the number of patients with criteria for a severe stenosis by additionally including patients with a milder degree of the disease without improving the predictive accuracy for aortic valve related events and cardiovascular death.

The percentage of patients with criteria for severe stenosis may differ depending on the population investigated. We therefore studied two distinct cohorts, one drawn from our institutional database representing typical patients referred to a tertiary care centre with a wide range of aortic stenosis severity as indicated by a mean jet velocity of $4.1 \mathrm{~m} / \mathrm{s}$ (range $2.5-6.7$ ). The other cohort was from the SEAS study comprising a highly selected population of patients with predominantly mild to moderate stenosis with a low cardiovascular risk profile as specified in the exclusion criteria of the trial. Despite the marked differences between the two study populations there was an almost identical pattern with respect to the increases seen in severe stenosis after calculation of $\mathrm{AVA}_{\text {index }}$.

In theory, calculation of $\mathrm{AVA}_{\text {index }}$ should eliminate body size dependency of AVA and not change the overall number of patients with severe stenosis in a given population but rather result in a decrease in the percentage in smaller and an increase in larger patients. The effect of indexation on the prevalence of severe stenosis in the two patient populations investigated in the present report, however, is quite different from the expected effect. Although indexation of AVA by BSA $\left(\mathrm{AVA}_{\text {index }}\right)$ clearly reduced body size dependency of AVA, using the currently accepted cut-off value of $0.6 \mathrm{~cm}^{2} / \mathrm{m}^{2}$ for $\mathrm{AVA}_{\text {index }}$ resulted in an increase of patients with criteria for severe stenosis over and above that seen with AVA (cut-off $<1.0 \mathrm{~cm}^{2}$ ) alone. Whereas small patients with a $\mathrm{BSA} \leq 1.75 \mathrm{~m}^{2}$ had no significant change in the prevalence of severe aortic stenosis, $\mathrm{AVA}_{\text {index }}$ resulted in an increase in the
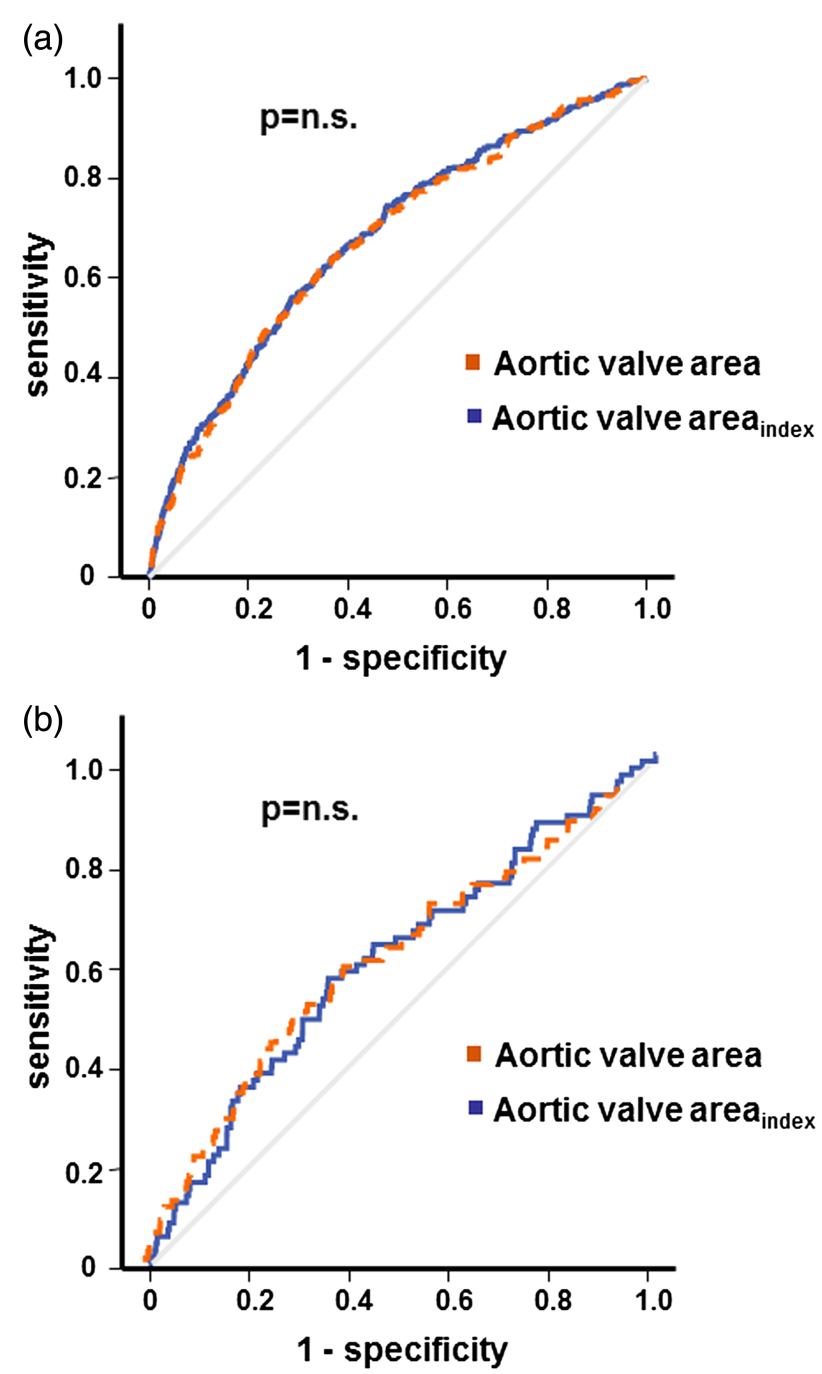

Figure 2 Predictive accuracy of echocardiographic parameters for valve related events. Receiver operating characteristic (ROC) curves illustrating the predictive accuracy of aortic valve area (AVA) and body surface area adjusted AVA ( AVA $\left._{\text {index }}\right)$ for aortic valve related events (a) and for cardiovascular death (b) over 46 months of follow-up in 1525 patients with mild to moderate aortic valve stenosis from the simvastatin and ezetimibe in aortic stenosis study. Area under the ROC curve was similar between AVA and AVA $_{\text {index }}$ (NS).

remaining patients (covering a BSA between 1.77 and $2.9 \mathrm{~m}^{2}$ ). These findings may in large part be explained by a discrepancy between partition values. The current relation between AVA and $\mathrm{AVA}_{\text {index }}$ is based on a BSA of $1.67 \mathrm{~m}^{2}$ (since 1.0/1.67 yields 0.6), whereas in the current study populations totalling 4368 patients the mean BSA was 1.89 and $1.91 \mathrm{~m}^{2}$, respectively.

\section{Special populations}

Even in the subgroups of 'very small' patients (BSA $<1.5$ or $\mathrm{BMI}<22$ ) there was only a trend towards a reduction of the percentage of severe stenosis, a finding potentially due to small sample size. Similarly, the increase in patients with severe stenosis seen with indexation was markedly more pronounced in males than in females, possibly due to smaller body size in the latter. Interestingly, in a recent retrospective Japanese study in 103 comparatively small asymptomatic patients with mean BSA of $1.5 \pm 0.2 \mathrm{~m}^{2}$, an $\mathrm{AVA}_{\text {index }}$ of $\geq 0.6 \mathrm{~cm}^{2} / \mathrm{m}^{2}$ was frequently observed in patients with an AVA $<1.0 \mathrm{~cm}^{2,5}$ and an $\mathrm{AVA}_{\text {index }}$ of $<0.6 \mathrm{~cm}^{2} / \mathrm{m}^{2}$ was predictive of a poor outcome. 
(a)

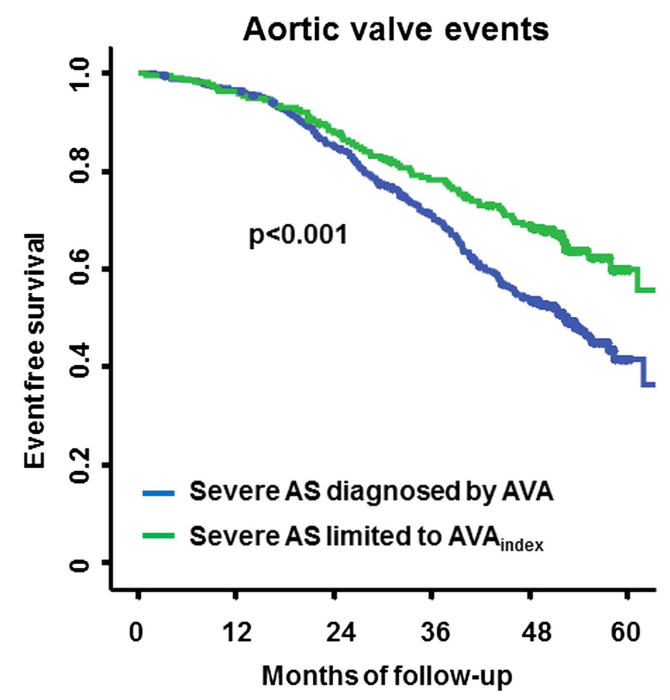

No. at risk

$\begin{array}{lllllll}\text { AVA }<1.0 \mathrm{~cm}^{2} & 470 & 453 & 395 & 323 & 241 & 10 \\ \begin{array}{c}\mathrm{AVA}_{\text {index }}<0.6 \mathrm{~cm}^{2} / \mathrm{m}^{2} \\ \left.\text { (and } A V A \geq 1.0 \mathrm{~cm}^{2}\right)\end{array} & 213 & 205 & 185 & 162 & 140 & 15\end{array}$

(b)

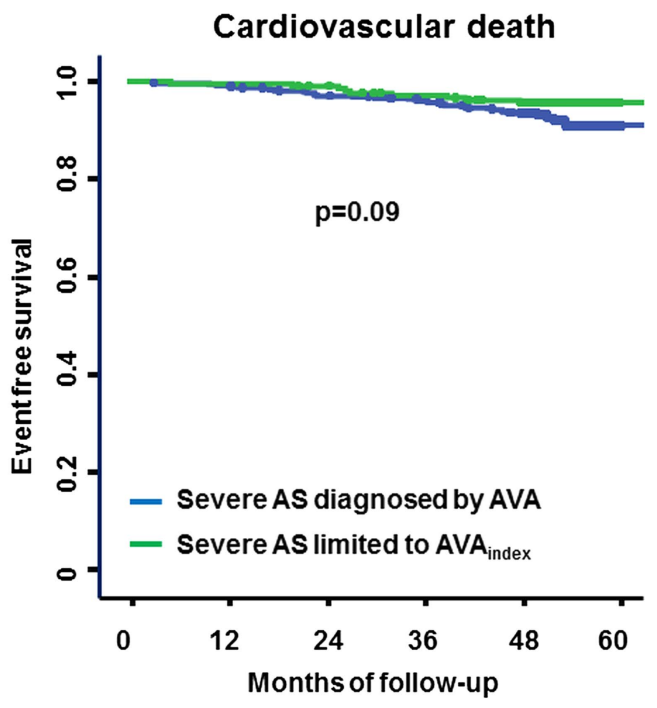

No. at risk

$\begin{array}{lllllll}A V A<1.0 \mathrm{~cm}^{2} & 470 & 453 & 395 & 323 & 241 & 10 \\ \begin{array}{c}A V A_{\text {index }}<0.6 \mathrm{~cm}^{2} / \mathrm{m}^{2} \\ \left(\text { and } A V A \geq 1.0 \mathrm{~cm}^{2} \text { ) }\right.\end{array} & 213 & 205 & 185 & 162 & 140 & 15\end{array}$

Figure 3 Outcome in patients additionally diagnosed with severe aortic stenosis (AS) due to the aortic valve area (AVA) index (AVA $\left.A_{\text {index }}\right)$ criterion. Kaplan-Meier plots illustrating significantly less aortic valve events in 213 patients additionally categorised as severe according and limited to the $\mathrm{AVA}_{\text {index }}$ criterion $\left(<0.6 \mathrm{~cm}^{2} / \mathrm{m}^{2}\right)$ compared to 470 patients with severe stenosis according to AVA $\left(<1.0 \mathrm{~cm}^{2}\right.$, $p<0.001)(a)$; together with a trend towards lower cardiovascular mortality $(p=0.09)(b)$.

On the other hand, the role of indexation of AVA by BSA in large patients has been challenged due to overcorrection by the potential influence of excess body weight. However, excluding patients with a $\mathrm{BMI} \geq 30$ did not change the pattern of increasing the prevalence of severe aortic stenosis in the predefined BSA quartiles. Therefore, the observed overcorrection in larger individuals is not a result of obesity.

Indexation has been recommended specifically in patients with the clinically challenging constellation of 'inconsistent grading' or 'low gradient severe aortic stenosis ${ }^{13}$ in whom AVA indicates severe stenosis $\left(<1.0 \mathrm{~cm}^{2}\right)$, however, mean pressure gradient remains below $40 \mathrm{~mm} \mathrm{Hg}$ despite normal ejection fraction and, therefore, does not fulfil the criterion of a severe stenosis. In theory, indexation should decrease not only the number of patients with severe stenosis but also the percentage of 'inconsistent grading' in small patients. In contrast, our results demonstrate that indexation of AVA in fact increases the overall number of patients with 'inconsistent grading' and does not reduce it even in the quartile with the smallest patients. Therefore, rather than improving the comparability of different parameters for the echocardiographic assessment of the severity of aortic stenosis, adjustment of AVA by BSA ( $\left(\mathrm{AVA}_{\text {index }}\right.$ ) with the current cut-off value of $<0.6 \mathrm{~cm}^{2} / \mathrm{m}^{2}$ augmented the already existing discrepancies in both populations investigated. However, adjusting the cut-off for severe stenosis to $\mathrm{AVA}_{\text {index }} 0.5 \mathrm{~cm}^{2} / \mathrm{m}^{2}$ as recently suggested, ${ }^{14}$ substantially decreased inconsistent grading, supporting the notion that adjustment to the proposed values may be appropriate.

\section{Predictive accuracy for clinical events}

In the SEAS population, $\mathrm{AVA}_{\text {index }}$ did not improve predictive accuracy for aortic valve events or cardiovascular death compared to AVA-neither in the whole population nor in patients with 'inconsistent grading' (data not shown)—despite successful elimination of body size dependency of AVA. Moreover, 213 patients in the SEAS cohort with severe aortic stenosis additionally diagnosed with and limited to the $\mathrm{AVA}_{\text {index }}$ criterion had a better clinical outcome than those with severe stenosis originally diagnosed with the AVA criterion, indicating that a cut-off value of $0.6 \mathrm{~cm}^{2} / \mathrm{m}^{2}$ for AVAindex increases the prevalence of severe aortic stenosis by including individuals with a milder degree of the disease.

\section{Clinical implications}

Severe aortic stenosis is currently defined by a peak velocity $>4.0 \mathrm{~m} / \mathrm{s}$, mean pressure gradient $>40 \mathrm{~mm} \mathrm{Hg}$, AVA $<1.0 \mathrm{~cm}^{2}$ and $\mathrm{AVA}_{\text {index }}<0.6 \mathrm{~cm}^{2} / \mathrm{m}^{2}$ in both European ${ }^{1}$ and North American guidelines. ${ }^{2}$ These different measures of stenosis severity should be interchangeable in the presence of normal flow conditions. However, recent studies have shown that AVA tends to overestimate stenosis severity in comparison to peak velocity and mean pressure gradient. ${ }^{15}{ }^{16}$ Furthermore, AVA introduces a strong correlation to body size, hindering the comparability of the different parameters used. The present study clearly shows that indexation of AVA by BSA reduces body size dependency of AVA. However, using the current cut-off value $\mathrm{AVA}_{\text {index }}<0.6 \mathrm{~cm}^{2} / \mathrm{m}^{2}$ results in a further increase in the number of patients with severe stenosis over and above that seen with AVA. Taken together our data demonstrate that the $\mathrm{AVA}_{\text {index }}$ criterion of $<0.6 \mathrm{~cm}^{2} / \mathrm{m}^{2}$ is too high, at least in predominantly Caucasian populations, and a cut-off $<0.5 \mathrm{~cm}^{2} / \mathrm{m}^{2}$ may be more appropriate. ${ }^{14}$ Importantly and as stated in the current guidelines, it should be kept in mind that 'absolute cut-off points cannot be relied upon for clinical decision-making and should be considered in combination with flow rate, pressure gradients, ventricular function, size and wall thickness, degree of valve calcification and blood pressure, as well as functional status'. ${ }^{1}$

\section{Limitations}

There are some limitations with this analysis. First, the calculations were performed on data from two mostly northern and western European patient populations and may not apply to populations with significantly different anthropometric characteristics. ${ }^{5}$ Second, alternative formulae for the assessment of BSA exist, 
however calculation of BSA based on work by Dubois and Dubois $^{17}$ did not change results significantly (data not shown). Third, the validity of linear adjustment of cardiac dimensions by BSA has been questioned ${ }^{18}$ and stratification of patients according to quartiles of BSA may therefore be an oversimplification with respect to the relationship between AVA and body size. Forth, overweight status may be a limitation of $\mathrm{AVA}_{\text {index }}{ }^{14}$ since BSA corrects not only for individual body size but also for acquired fat tissue. The adequate method to correct for excess body weight has not been clarified yet, but obesity could have contributed to the increased prevalence of severe aortic stenosis in large individuals. However, restricting the analysis to normal weight patients retained the pattern of increasing prevalence of severe aortic stenosis by $\mathrm{AVA}_{\text {index }}$ compared to AVA.

\section{CONCLUSION}

Indexing AVA by BSA and applying the current partition value of $\mathrm{AVA}_{\text {index }}<0.6 \mathrm{~cm}^{2} / \mathrm{m}^{2}$ for severe stenosis increases the prevalence of severe aortic valve stenosis compared to unindexed AVA by including individuals with a milder degree of disease without improving the predictive accuracy for clinical events.

\author{
Author affiliations \\ ${ }^{1}$ University Heart Centre Freiburg/Bad Krozingen, Bad Krozingen, Germany \\ ${ }^{2}$ Department of Cardiology, Asklepios Clinic St Georg, Hamburg, Germany \\ ${ }^{3}$ Institute of Medicine, University of Bergen, Haukeland University Hospital, \\ Bergen, Norway \\ ${ }^{4}$ Department of Medicine, Skellefteå, Sweden/Institution of Public Health and Clinical \\ Medicine, Umeå University, Umeå, Sweden \\ ${ }^{5}$ Cardiothoracic Centre, Guys-St Thomas Hospital Trust, London, UK \\ ${ }^{6}$ Department of Medical Research, OUH Svendborg Hospital, Svendborg, Sweden \\ ${ }^{7}$ Medizinische Klinik I, Universitätsklinikum Rostock, Rostock, Germany \\ ${ }^{8}$ Ullevål, Centre of Preventive Medicine, University of Oslo, Oslo, Norway \\ ${ }^{9}$ Department of Cardiology, University Hospitals of South Manchester, \\ Manchester, UK \\ ${ }^{10}$ Division of Cardiology, Oslo University Hospital, Aker, Norway \\ ${ }^{11}$ Heart Health Group and Lund University, Malmö, Sweden \\ ${ }^{12}$ Department of Cardiology, Rigshospitalet, Copenhagen, Denmark \\ ${ }^{13}$ Department of Cardiology, University Hospital Basel, Basel, Switzerland
}

Contributors JM as corresponding author acts as guarantor. NJ, CGB and JM were involved in the study conception and design; acquisition, analysis and interpretation of data; drafted the article and had final approval of the manuscript. RK was involved in the study design, data acquisition and interpretation of data. $E B, E G, K B$, $J B C, K E, C A N, T R J, S R, A B R, R W, K W$ and FN were involved in interpretation of data, revising the article, and final approval of the manuscript.

Competing interests None.

Provenance and peer review Not commissioned; externally peer reviewed.

Data sharing statement No additional unpublished data are available.

\section{REFERENCES}

1 Authors/Task Force M, Vahanian A, Alfieri O, et al. Guidelines on the management of valvular heart disease (version 2012): The Joint Task Force on the Management of Valvular Heart Disease of the European Society of Cardiology (ESC) and the European Association for Cardio-Thoracic Surgery (EACTS). Eur Heart $J$ 2012;33:2569-619.

2 Bonow RO, Carabello BA, Chatterjee K, et al. 2008 Focused update incorporated into the ACC/AHA 2006 guidelines for the management of patients with valvular heart disease: a report of the American College of Cardiology/American Heart Association Task Force on Practice Guidelines (Writing Committee to Revise the 1998 Guidelines for the Management of Patients With Valvular Heart Disease): endorsed by the Society of Cardiovascular Anesthesiologists, Society for Cardiovascular Angiography and Interventions, and Society of Thoracic Surgeons. Circulation 2008;118:e523-661.

3 Rahimtoola SH. The problem of valve prosthesis-patient mismatch. Circulation 1978;58:20-4.

4 Baumgartner $\mathrm{H}$, Hung J, Bermejo J, et al. Echocardiographic assessment of valve stenosis: EAE/ASE recommendations for clinical practice. Eur J Echocardiogr 2009;10:1-25.

5 Saito T, Muro T, Takeda $\mathrm{H}$, et al. Prognostic value of aortic valve area index in asymptomatic patients with severe aortic stenosis. Am J Cardiol 2012;110:93-7.

6 Rossebo $A B$, Pedersen TR, Boman $K$, et al. Intensive lipid lowering with simvastatin and ezetimibe in aortic stenosis. N Eng/ J Med 2008;359:1343-56.

7 Evangelista A, Flachskampf $F$, Lancellotti $P$, et al. European Association of Echocardiography recommendations for standardization of performance, digital storage and reporting of echocardiographic studies. Eur J Echocardiogr 2008;9:438-48. Epub 2008/06/27.

8 Galderisi M, Henein MY, D'Hooge J, et al. Recommendations of the European Association of Echocardiography: how to use echo-Doppler in clinical trials: different modalities for different purposes. Eur J Echocardiogr 2011;12:339-53.

9 Rossebo $A B$, Pedersen TR, Allen $C$, et al. Design and baseline characteristics of the simvastatin and ezetimibe in aortic stenosis (SEAS) study. Am J Cardiol 2007:99:970-3.

10 Minners J, Allgeier M, Gohlke-Baerwolf C, et al. Inconsistencies of echocardiographic criteria for the grading of aortic valve stenosis. Eur Heart J 2008;29:1043-8.

11 Mosteller RD. Simplified calculation of body-surface area. N Engl J Med 1987;317:1098.

12 DeLong ER, DeLong DM, Clarke-Pearson DL. Comparing the areas under two or more correlated receiver operating characteristic curves: a nonparametric approach. Biometrics 1988;44:837-45.

13 Dumesnil JG, Pibarot P, Carabello B. Paradoxical low flow and/or low gradient severe aortic stenosis despite preserved left ventricular ejection fraction: implications for diagnosis and treatment. Eur Heart J 2010;31:281-9.

14 Michelena HI, Margaryan E, Miller FA, et al. Inconsistent echocardiographic grading of aortic stenosis: is the left ventricular outflow tract important? Heart 2013;99:921-31

15 Minners J, Allgeier M, Gohlke-Baerwolf C, et al. Inconsistent grading of aortic valve stenosis by current guidelines: haemodynamic studies in patients with apparently normal left ventricular function. Heart 2010;96:1463-8.

16 Jander N, Minners J, Holme I, et al. Outcome of patients with low-gradient 'severe' aortic stenosis and preserved ejection fraction. Circulation 2011;123:887-95.

17 Wang Y, Moss J, Thisted R. Predictors of body surface area. J Clin Anesth 1992:4:4-10.

18 Neilan TG, Pradhan AD, Weyman AE. Derivation of a size-independent variable for scaling of cardiac dimensions in a normal adult population. J Am Soc Echocardiogr 2008:21:779-85. 